\title{
Biocompósitos de Poliuretano reforçados com Fibras de Coco Verde
}

\section{Polyurethane reinforced with green coconut fiber biocomposites}

\author{
Wagner Martins Florentino ${ }^{1}$ \\ Amanda Brandão ${ }^{2}$ \\ Patrícia Câmara Miléo ${ }^{3}$ \\ Shane Aparecida Soares Goulart ${ }^{4}$ \\ Daniella Regina Mulinari ${ }^{5}$
}

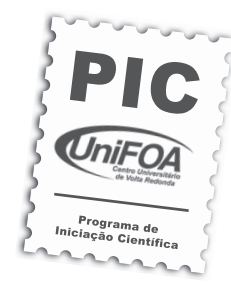

\section{Artigo \\ Original \\ Original \\ Paper}

\section{Palavras-chave:}

Fibras de coco verde

Tratamento alcalino

Propriedades

mecânicas

\section{Resumo:}

O estudo e desenvolvimento de materiais biodegradáveis e de fontes renováveis têm aumentado nos últimos anos. Neste contexto, os biocompósitos poliméricos com fibras naturais surgem como uma boa alternativa para novas aplicações. No entanto, para que as fibras naturais e a matriz polimérica atuem em uma determinada aplicação, o contato interfacial entre elas deve ser adequado. Portanto, o objetivo deste trabalho foi estudar o efeito da modificação química das fibras de coco verde através do tratamento alcalino $(\mathrm{NaOH})$ no desenvolvimento de biocompósitos de poliuretano. A modificação das fibras foi avaliada pela técnica de microscopia eletrônica de varredura. Também foram avaliadas as propriedades mecânicas dos compósitos PU reforçados com as fibras de coco in natura e modificadas.

\section{Abstract}

In recent years the study and development of biodegradable materials and renewable sources have increased. In this context, the polymer composites reinforced with natural fibers has being presented as a good alternative to the new applications. However, in order to have a determined application, the interfacial contact between the natural fibers and the polymer matrix must be appropriate. Therefore, the aim of this work was to study the effect of the chemical modification in the green coconut fibers through the alkali treatment $(\mathrm{NaOH})$ in the polyurethane composites development. The fibers modification was analyzed by scanning electron microscopy. The mechanical properties of PU composites reinforced with green coconut fibers in nature and modified ones were also analyzed.
\end{abstract}

Key words:

Green coconut fibers

Alkali treatment

Mechanical properties

\footnotetext{
${ }^{1}$ Graduado em Ciências Biológicas - UniFOA

${ }^{2}$ Discente do $6^{\circ}$ período de Ciências Biológicas - UniFOA

${ }^{3}$ Doutoranda em Biotecnologia Industrial - Escola de Engenharia de Lorena /USP

${ }^{4}$ Docente do Curso de Engenharia Ambiental - Centro Universitário de Volta Redonda- UniFOA

${ }_{5}^{5}$ Docente do Mestrado Profissional em Materiais - MEMAT - Centro Universitário de Volta Redonda- UniFOA
}

Recebido em $03 / 2011$

Aprovado em $12 / 2011$ 


\section{Introdução}

A produção de resíduos oriundos do coco de agroindústrias é extremamente significativa, provocando grandes problemas com relação ao meio ambiente para o descarte do mesmo (PANNIRSELVAM et al., 2007). E como essas fibras resistem a temperaturas de até $200^{\circ} \mathrm{C}$, torna-se bastante atraente para utilização como reforço em compósitos. Essas fibras são constituídas de materiais lignocelulósicos, obtidos do mesocarpo do coco (Cocos nucifera) (ARAÚJO, 2003).

Nos últimos anos, o uso de fibras naturais como reforço em materiais poliméricos teve um acelerado crescimento, devido a algumas características das fibras, tais como, ser proveniente de fonte renovável, apresentar baixo custo, serem biodegradáveis, recicláveis e não tóxicas (ARAÚJO, 1998; MOTHÉ; ARAUJO, 2004). Portanto essas fibras têm sido utilizadas como reforço em polímeros visando à substituição das fibras sintéticas (KAPULSKIS et al., 2005).

No entanto, para que as fibras naturais e a matriz polimérica atuem conjuntamente em uma determinada aplicação, o contato interfacial entre elas tem que ser adequado. Dessa forma, inúmeros tratamentos físicos e químicos têm sido realizados para melhorar a adesão entre fibras naturais e matrizes poliméricas (ALMEIDA et al., 2005; TSERKI et al., 2005).

Estudos indicam que as modificações químicas realizados nas fibras naturais influenciam no comportamento mecânico dos biocompósitos (WAMBUA et al., 2003).

Os Poliuretanos (PUs) são polímeros pertencentes à classe dos termorrígidos e podem ser obtidos pela combinação de diversas moléculas mais simples, sendo que os monômeros mais utilizados são os isocianatos e polióis, dando assim origem a compostos com a chamada ligação uretana.

O Brasil produz anualmente cerca de 335 mil toneladas de poliuretano e, até 2014, esse número pode chegar a 441 mil toneladas, apresentando uma evolução média de $4,7 \%$ ao ano. Tamanha produção gera mensalmente ao país 2,5 mil toneladas de resíduos de poliuretano. Os destinos desses restos normalmente são a queima indiscriminada e os aterros industriais, que levam milhares de anos para se decompor.
Além disso, a produção de poliuretano utiliza principalmente compostos derivados de petróleo, o que tem se tornado um problema ambiental por se tratar de um recurso não renovável (AKRAM et al., 2009).

Pesquisas vêm sendo feitas no intuito de utilizar matérias-primas renováveis na produção de poliuretanos, como óleos vegetais, evitando-se assim, o consumo de derivados do petróleo, além de gerar polímeros biodegradáveis. Para síntese desses biopolímeros, têm-se utilizado óleos vegetais como o óleo de mamona em vez de polióis a base de petróleo, e bons resultados estão sendo obtidos (PANNIRSELVAM et al., 2007). Atualmente o biopolímero de poliuretano pode ser produzido a partir do óleo vegetal da mamona -Ricinus communis, apresentando grande biocompatibilidade e boas propriedades químicas e físicas (CLEMONS, 2002; FENGEL; WEGENER, 1999).

A produção de biocompósitos também recebe grande interesse de pesquisadores. Biopolímeros como o próprio poliuretano derivado de óleo de mamona podem servir de matrizes para compósitos reforçados com fibras vegetais.

Estudos indicam que as modificações químicas realizados nas fibras naturais influenciam no comportamento mecânico dos biocompósitos (KEENER et al., 2004).

Portanto o objetivo deste trabalho foi avaliar o efeito da modificação química das fibras de coco verde por meio do tratamento alcalino $(\mathrm{NaOH})$ nas propriedades mecânicas dos compósitos poliuretano, reforçados com fibras de coco verde.

\section{Materiais e Métodos}

\subsection{Obtenção das Fibras de Coco Verde}

As fibras de coco foram obtidas através do Projeto Coco Verde (Rio de Janeiro). Posteriormente as fibras foram cortadas em tamanhos de aproximadamente 5 centímetros e moídas em um moinho de facas. Em seguida as fibras foram peneiradas em uma peneira de 25 mesh. 


\subsection{Tratamento Alcalino na Fibra de Coco Verde}

O tratamento superficial empregado nas fibras de coco foi o tratamento alcalino com soluções de $\mathrm{NaOH}$. Nesse tratamento, $10 \mathrm{~g}$ de fibras de coco foram adicionados em um erlenmeyer, contendo $100 \mathrm{~mL}$ da solução de hidróxido de sódio a $10 \% \mathrm{~m} / \mathrm{m}$ sob agitação constante por 1 hora. Decorrido o tempo de tratamento, a solução foi filtrada e lavada com água destilada até que a água resultante da filtragem apresentasse o $\mathrm{pH}$ neutro. Em seguida, as fibras foram colocadas em uma placa de Petri as quais foram secas em uma estufa à $60^{\circ} \mathrm{C}$ por 24 horas.

\subsection{Análise da modificação das fibras}

As fibras de coco in natura e tratadas foram caracterizadas por Microscopia eletrônica de varredura (MEV). As micrografias foram obtidas em um microscópio eletrônico de varredura JEOL JSM5310, disponível no Instituto Nacional de Pesquisas Espaciais/ INPE em São José dos Campos, operando de 15 a $20 \mathrm{kw}$ e utilizando detector de elétrons secundários. As amostras foram fixadas em um suporte com auxílio de uma fita de carbono autocolante dupla face e submetidas ao recobrimento metálico com ouro.

\subsection{Preparação dos Compósitos de Poliuretano com Fibra de Coco}

Os compósitos de poliuretano reforçado com fibras de coco foram obtidos por meio do processo de moldagem por compressão. Para a expansão do poliuretano à base de óleo de mamona, os reagentes foram misturados conforme a relação estequiométrica de $1,5: 1$, em massa, de poliol e diisocianato, respectivamente. Os reagentes foram misturados em um copo descartável e, em seguida, foi adicionado fibra de coco $10 \% \mathrm{~m} / \mathrm{m}$ tratada em solução alcalina por 2 minutos e vertido em um molde. Após o vertimento do material, o molde foi pressionado por grampos por um período de 24 horas. O molde foi pressionado por duas placas de vidro as quais foram protegidas com folhas plásticas que auxiliaram a desmoldagem dos corpos-de-prova. Esse procedimento foi realizado com os compósitos de poliuretano com fibras de coco in natura, fibras tratadas e o poliuretano puro.

\subsection{Ensaios Mecânicos}

Os ensaios de tração dos compósitos e do poliuretanopuroforamrealizadosnoLaboratório de Ensaios Mecânicos do Departamento de Mestrado em Materiais do Centro Universitário de Volta Redonda - UniFOA, em um equipamento da marca EMIC modelo DL10.000 a uma velocidade de $5 \mathrm{~mm} \cdot \mathrm{min}^{-1}$, com dimensões de acordo com a norma ASTM D 638 - 03 com $13 \mathrm{~mm}$ de largura, $165 \mathrm{~mm}$ de comprimento e 3 mm de espessura.

\section{Resultados e Discussão}

\subsection{Microscopia Eletrônica de Varredura (MEV)}

As micrografias obtidas por MEV permitiram avaliar os aspectos morfológicos das fibras. As superfícies das fibras do coco in natura e tratadas apresentaram características diferentes. A Fig. 1 evidencia as micrografias das fibras de coco in natura obtidas em diferentes ampliações. 


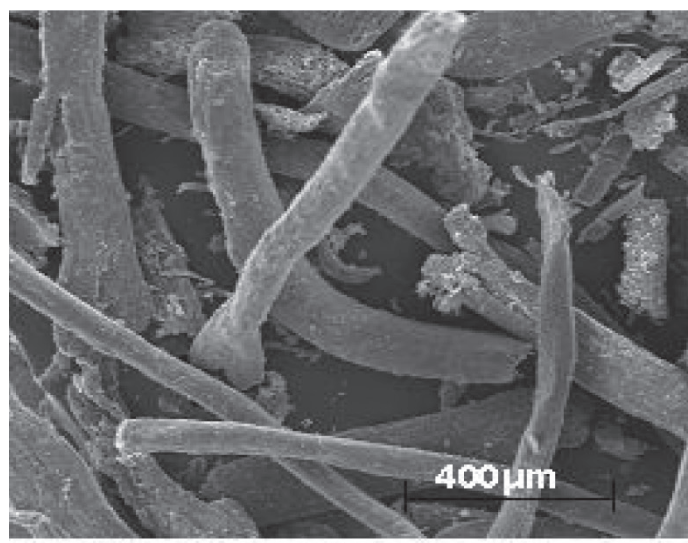

(a)

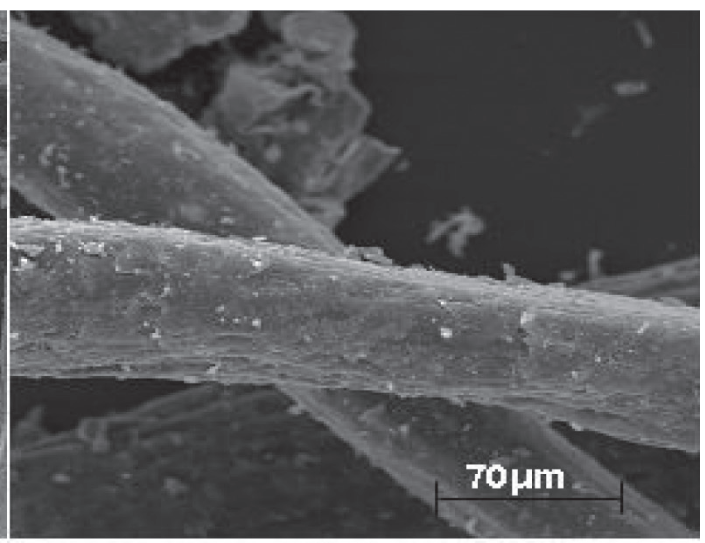

(b)

Figura 1 - Micrografias das fibras de coco in natura: (a) 100X; (b) 500X;

Nas micrografias das fibras de coco in natura (Fig. 1a e 1b) observou-se uma superfície lisa e a presença de extrativos cerosos, envolvendo toda a superfície da fibra quando comparado com as fibras tratadas (Fig. 2).

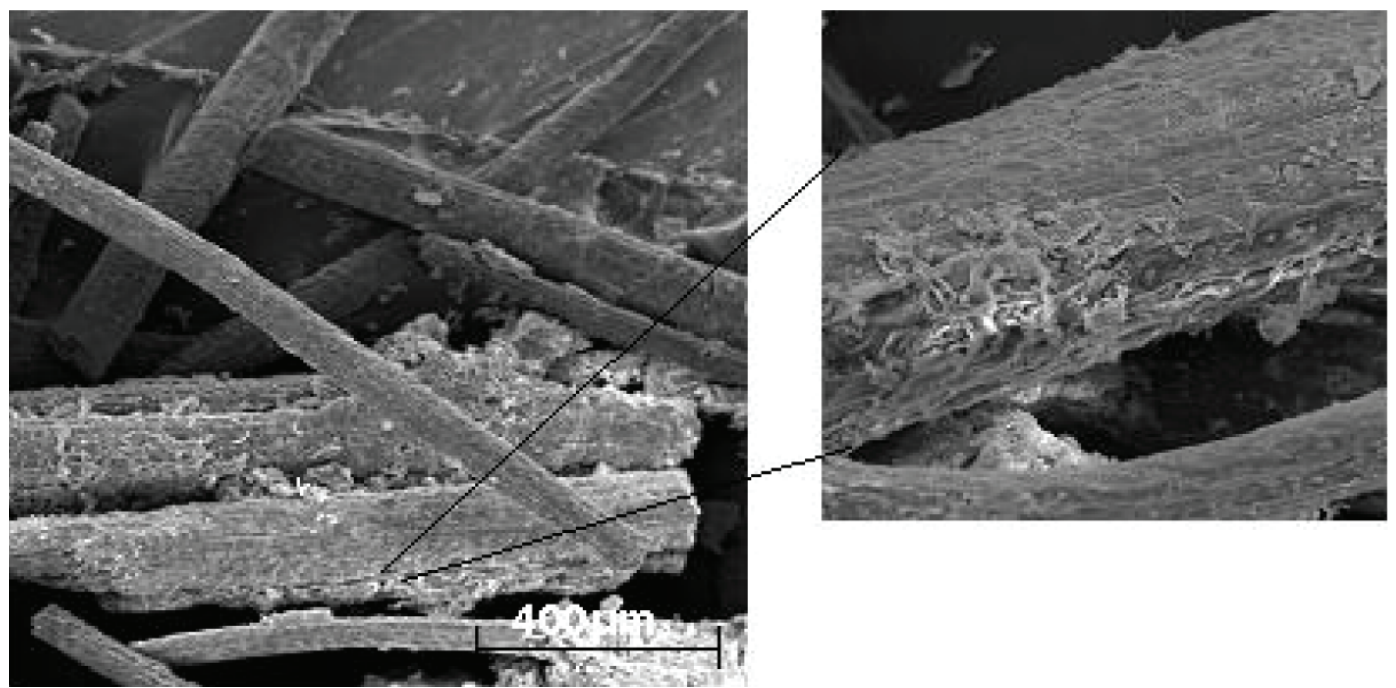

Figura 2 - Micrografias das fibras de coco tratadas 100X.

Na Fig. 2 foi verificado que o tratamento alcalino removeu com eficácia os extrativos que envolviam a fibra, permitindo a visualização das fibrilas e maior empacotamento das cadeias de celulose. As ceras e graxas que envolvem as fibras naturais impermeabilizam a celulose, diminuindo a área de adesão entre a matriz e o reforço, sua remoção é de fundamental importância para o aumento das propriedades mecânicas dos compósitos.

\subsection{Ensaio de Tração}

A Tab. 1 mostra os valores do limite de resistência à tração obtida dos ensaios mecânicos das misturas preparadas com diferentes tipos de fibras. 
Tabela 1 - Valores médios dos ensaios em tração nas fibras de coco

\begin{tabular}{c|c|c|c}
\hline Materiais & PU puro & $\begin{array}{c}\text { PU/FC in } \\
\text { natura }\end{array}$ & $\begin{array}{c}\text { PU/FC } \\
\text { tratadas }\end{array}$ \\
\hline Limite de resistência à tração (MPa) & $\mathbf{0 , 4 0}$ & $\mathbf{0 , 5 8}$ & $\mathbf{0 , 8 6}$ \\
\hline Desvio padrão & 0,05 & 0,11 & 0,13 \\
\hline
\end{tabular}

Analisando-se os resultados dos compósitos e do poliuretano puro, observouse um aumento na resistência do material. Comparando-se a resistência dos compósitos reforçados com fibras de coco in natura e modificadas, foi observado também que o tratamento utilizado nas fibras influenciou significativamente na resistência do material.

\section{Referências Bibliográficas}

1. AKRAM, T.; MEMON, S. A.; OBAID, H. Production of low cost self compacting concrete using bagasse ash. Construction and Building Materials, Amsterdã, v.23, p.703-712, 2009.

2. AlMEIDA, A. L. F. S.; CALADO, V.; BARRETO, D. W.; ALMEIDA, J. R. M. Acetilação da fibra de bucha (Luffa cylindrica). Polímeros: ciência e tecnologia. São Carlos, v.65, p. 59-62, 2005.

3. ARAÚJO, C. R. Cinética de Decomposição Térmica de Compósitos Poliméricos com Fibras de Curauá, Tese de Doutorado, (Doutorado em Química) - UFRJ, Universidade Federal do Rio de Janeiro, Rio de Janeiro, Brasil, 2003.

4. ARAÚJO,C.R.Compósitos Elastoméricos de Poliuretanos com Fibras Naturais, Tese de Mestrado, (Mestrado em Química)UFRJ, Universidade Federal do Rio de Janeiro, Rio de Janeiro, Brasil, 1998.

5. CLEMONS, C. Wood-plastics composites in the United States: the interfacing of two industries. Journal Forest Products, v.52, p.10-18, 2002.

\section{Conclusões}

Com base nos resultados obtidos foi possível concluir que o tratamento superficial com solução alcalina de $\mathrm{NaOH}$ foi satisfatória para o aumento das resistência à tração nos compósitos. Esse resultado pode ser explicado pela remoção de extrativos e ceras, as quais favoreceram a interação entre fibra/matriz.

6. FENGEL, D.; WEGENER, G. WoodChemistry, Ultrastructure, Reactions. European JOURNAL OF WOOD AND WOOD PRODUCTS, New York, v.42, p. 188-190, 1999.

7. KAPULSKIS, T. A.; DE JESUS, R. C.; MEI, L. H. I. Modificação Química de Fibras de Coco e de Sisal Visando Melhorar suas Interações Interfaciais com Matrizes Poliméricas Biodegradáveis. Anais VI Congresso Brasileiro de Engenharia Química em Iniciação Científica, Campinas, v.6, p.1-6, 2005.

8. KEENER, T. J.; STUART, R. K.; BROWN, T. K. Maleated coupling agents for natural fibre composites. Composites: Part A, New York v.35, p.357-362, 2004. MOTHÉ, C. G.; ARAUJO, C. R. Caracterização Térmica e Mecânica de Compósitos de Poliuretano com Fibras de Curauá. Polímeros: Ciência e Tecnologia, Rio de janeiro, v. 14, p. 274-278, 2004.

9. PANNIRSELVAM, P. V.; LIMA, F. A. M.; DANTAS, B. S.; SANTIAGO, B. H. 
10. S. Desenvolvimento de Projeto para Produção de Fibra de Coco com Inovação de Tecnologia Limpa e Geração de Energia. Revista Analytica, Campinas, v. 15, p. 56-62, 2007.

11. TSERKI, V.; ZAFEIROPOULOS, N. E.; SIMON, F.; PANAYIOTOU, C. A. Study of the effect of acetylation and propionylation surface treatments on natural fibres. Composites: Part A: applied science and manufacturing, New York, v.43, p. 1110-1118, 2005.

12. WAMBUA, P.; IVENS J.; VERPOEST, I. Natural fibres: Can they replace glass in fibre reinforced plastics. Composites Science and Technology, Amsterdã, v.61, p. 1259-1264, 2003.

Endereço para Correspondência:

Daniella Regina Mulinari

daniella.mulinari@foa.org.br

Centro Universitário de Volta Redonda - UniFOA

Departamento de Engenharia - UniFOA

Campus Universitário Olezio Galotti

Av. Paulo Erley Abrantes, 1325

Três Poços - Volta Redonda - RJ

CEP: $27.240-560$

Informações bibliográficas:

Conforme a NBR 6023:2002 da Associacão Brasileira de Normas Técnicas (ABNT), este texto científico publicado em periódico eletrônico deve ser citado da seguinte forma: FLORENTINO, Wagner Martins; BRANDÃO, Amanda; MILÉO, Patrícia Câmara; GOULART, Shane Aparecida Soares; MULINARI, Daniella Regina. Biocompósitos de Poliuretano reforçados com Fibras de Coco Verde. Cadernos UniFOA. Volta Redonda, Ano VI, n. 17, dezembro 2011. Disponível em: <http://www.unifoa.edu.br/cadernos/ 